\title{
Development of 3D Color Space to Improve Learning of Color Theory
}

\author{
$\mathrm{Ng}$ Woon Lam, Xiao Yikai \\ School of Art, Design \& Media, Nanyang Technological University \\ 81 Nanyang Drive, Room 2-10, Singapore 637458 \\ ngwoonlam@ntu.edu.sg, xiao0067@e.ntu.edu.sg
}

Keywords: Munsell, Color, Space, 3D.

\begin{abstract}
Munsell Color System is a framework of practical color theory commonly adopted by the industry. However, it has not been well adopted in the area of art education at tertiary institutions teaching color theory [1]. The popular model used in current art schools is the Itten Color Wheel [2]. A paper was published to introduce Munsell Color System for color theory training for art students [3]. The Munsell Color System is a 3D model; therefore, visualization is not as direct as a 2D Itten Color Wheel. The objective is to develop a 3D visualization software and color analytical tool to assist students' learning of Munsell Color System as well as allowing self-driven learning through a new color analytical tool for analysis of color scheme of important and relevant artworks like masterpieces and students' artworks.
\end{abstract}

\section{Introduction}

Color theory training is one critical aspect of visual artists. Current training in most tertiary art institutions is based on the Itten Color Wheel [2]. The Itten Color Wheel is a symmetrical 2D model. With the symmetrical model, it assumes practical similarity between all hues. However, in reality as shown by a practical 3D model, the Munsell Color Space, each hue is practically different in range of tone it may stretch through [4]. Therefore, the practical range of each hue is expected to be very different from each other. Hence, a 3D practical model like Munsell Color Space, formed by current available pigments is the closest to the field of practice. A study was carried out to compare students' response to the Itten Color Wheel against Munsell Color Space [3]. Although students like the comprehensiveness of the Munsell Color Space, as it was able to show the relative browns and gray colors of their respective pure hue, it was difficult for them to visualize a 3D model. For example, yellow ochre is brown that was nearer to yellow, therefore it was located near to pure yellow hue on a Munsell Color Space. However, to visualize and remember a 3D model for practical usage, was a challenge to students. To resolve this issue, this project will create a 3D software model of the Munsell Color Space to ease students' difficulty in visualizing the model. In addition, the 3D model developed will incorporate a color analytical tool for students to carry study of any color images.

The development process consists of five major phases: project initial phase, project design phase, project implementation, project testing and evaluation phase. The main development tool for this application is Eclipse (Java IDE) and 3ds Max (3D modelling tool). Eclipse is used as the main programming software. It serves as the control of user interface, data processing and interacting with 3ds Max while 3ds Max serves the purpose of presenting the 3D Model of Munsell Color Space. 3ds Max reduces the developing time to create a complete 3D model from scratch while its limitation will be discussed.

The application contains two main functions. The first one is based on the 3D model of the Munsell Color Space, through which the basic information about the Munsell color framework will be presented. The other one is to use the mapping algorithm to map the colors appeared in the art pieces to the built model and then use it to analyse different color schemes of either masterpieces or any artworks relevant to students' learning. 


\section{Development of Software}

The development of the software was separated to two stages. Stage 1 was to gathering information about Munsell Color System. In a Munsell Color Space, the three attributes of colors

In Munsell Color Space, colors are specified based on three different color attributes: hue $(\mathrm{H})$, value ( $\mathrm{V}$, indicating lightness), and chroma $(\mathrm{C}$, indicating color purity). Therefore, it is also referred to as HVC color system. It was created by Professor Albert H. Munsell in the 1910s [5]. The system consists of three independent dimensions which can be represented cylindrically in three dimensions as an asymmetrical color solid.

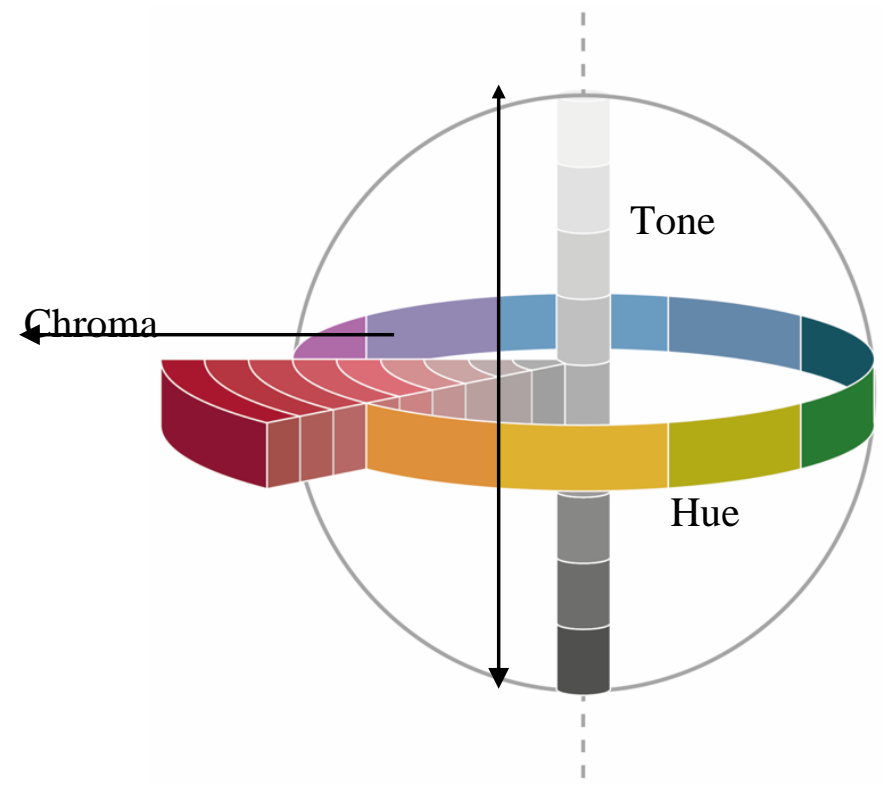

Figure 1. The Munsell Color Space and three color attributes [5].

The asymmetrical representation of color space is practically more accurate than the Itten Color Wheel which presents only prismatic colors based a two-dimensional format. On the Munsell Color Space the hue of each color is measured by degrees around horizontal circles. Each horizontal circle is divided into five principal hues: $\operatorname{Red}(\mathrm{R})$, Yellow $(\mathrm{Y})$, Green $(\mathrm{G}), \operatorname{Blue}(\mathrm{B})$ and Purple(P). Each primary color can mix with the adjacent colors to obtain a continuous variation from one to the other. For example, red and yellow may be mixed in any proportion to obtain all the hues red through orange to yellow(YR). The same is said of yellow and green(GY), green and blue(BG), blue and purple(PB), and purple and red(RP). This series returns to the starting point, so it can be arranged around a circle. Munsell placed them at equal intervals around a circle. For simplicity, he used the initials as symbols to designate the ten hue sectors: R, YR, Y, GY, G, BG, B, PB, P, and RP. Each of these 10 steps, is then broken into 10 sub-steps, so that 100 hues are given integer values (e.g. from $1 \mathrm{R}, 2 \mathrm{R} \ldots 10 \mathrm{R}$ ). In practice, color charts conventionally specify 40 hues, in increments of 2.5 (e.g. $2.5 \mathrm{R}, 5 \mathrm{R}, 7.5 \mathrm{R}$, 10R).

These Munsell notation of colors need to be converted to an sRGB value so that computer can use the values to plot the 3D model. The conversion is based on an available system [6] previously done by Paul Centore in 2013. 
Table 1. The conversion for hue 5Y with different. Each set of V,C and sRGB represents Value (V) and Chroma (C) of 5Y (Yellow); while sRGB number set, represents the converted number for software programming purpose.

\begin{tabular}{|c|c|c|c|c|c|c|c|c|}
\hline $\mathrm{V}$ & $\mathrm{C}$ & sRGB & V & $\mathrm{C}$ & sRGB & $\mathrm{V}$ & $\mathrm{C}$ & sRGB \\
\hline 3 & 2 & {$[80,72,52]$} & 6 & 6 & {$[170,148,69]$} & 8 & 4 & {$[217,202,146]$} \\
\hline 4 & 2 & {$[105,97,73]$} & 6 & 8 & {$[175,147,35]$} & 8 & 6 & {$[223,202,119]$} \\
\hline 4 & 4 & {$[112,96,49]$} & 7 & 2 & {$[184,175,148]$} & 8 & 8 & {$[228,202,90]$} \\
\hline 5 & 2 & {$[131,123,98]$} & 7 & 4 & {$[190,175,121]$} & 8 & 10 & {$[233,201,54]$} \\
\hline 5 & 4 & {$[138,122,73]$} & 7 & 6 & {$[197,175,94]$} & 9 & 2 & {$[238,230,197]$} \\
\hline 5 & 6 & {$[144,121,43]$} & 7 & 8 & {$[202,174,65]$} & 9 & 4 & {$[245,230,169]$} \\
\hline 6 & 2 & {$[157,149,122]$} & 7 & 10 & {$[206,174,15]$} & 9 & 6 & {$[251,230,141]$} \\
\hline 6 & 4 & {$[164,149,97]$} & 8 & 2 & {$[211,202,172]$} & 9 & 8 & {$[255,230,112]$} \\
\hline
\end{tabular}

All values on the Munsell Color Space are converted to sRGB number sets and stored in SQL database for retrieval. These datasets can be retrieved for plotting a 3D model of the Munsell Color Space as shown Figure 2, or used to analyse any image color scheme mapped against this Munsell Color Space in sRGB number sets.

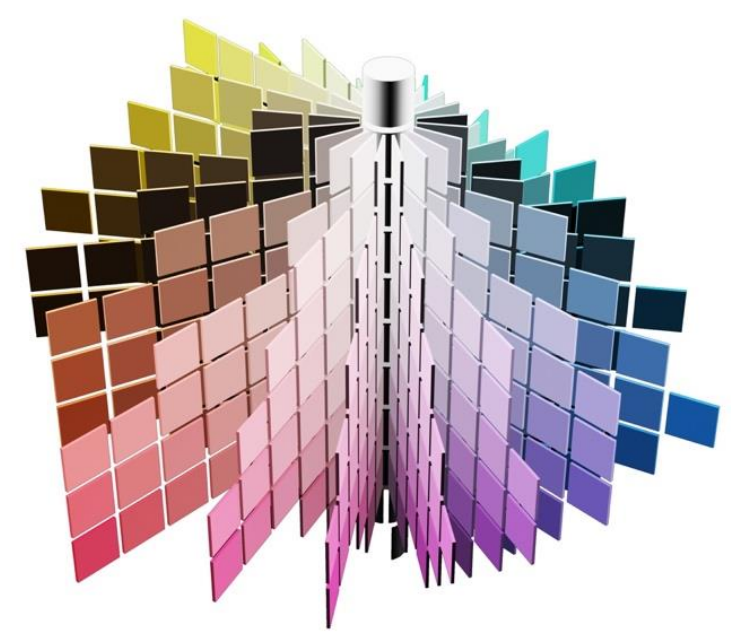

Figure 2. A 3D model of the Munsell Color Space plotted with converted sRGB number sets through 3ds Max software, called by Java programming as a Java Import.

When an image is loaded for analysis, each pixel on the image is read and stored as an sRGB number set. Again, all the number sets forming new array is then stored for comparison. Since the Munsell Color Space does not consist all the pixel sRGB numbers, during the mapping process, the closest neighbour will be used. At the end of the process, the color scheme of the analysed image can be mapped as a new 3D model to represent how the colors are used in that image. This could assist students' learning of colors in three ways.

First, students are able to visualize the region on colors being used for that image. If a series of images of one specific master artist is analysed, students are able to see if there is a common scheme of colors being used by that master artist. Since the process of analysis is a lot faster than manually mapping an image, this speed up students' analysis of master artworks or any other set of artworks that students wish to understand their color application logics.

The second advantage is for students' understanding of historical development of color application. The color application has relevant connection with the human history and scientific progress as well. The evidence could be seen through comparing artworks done during the Renaissance and artworks 
done during the Impressionist period. The important event that instilled the huge difference in color application was the Industrial Revolution. It enabled manufacturing of paint pigments in tube in bulk quantity with ease. In addition, Chemistry development during the same period also expanded the range of available color pigments. Therefore, the Impressionists were able to bring convenient tube paints out and work on location [7].

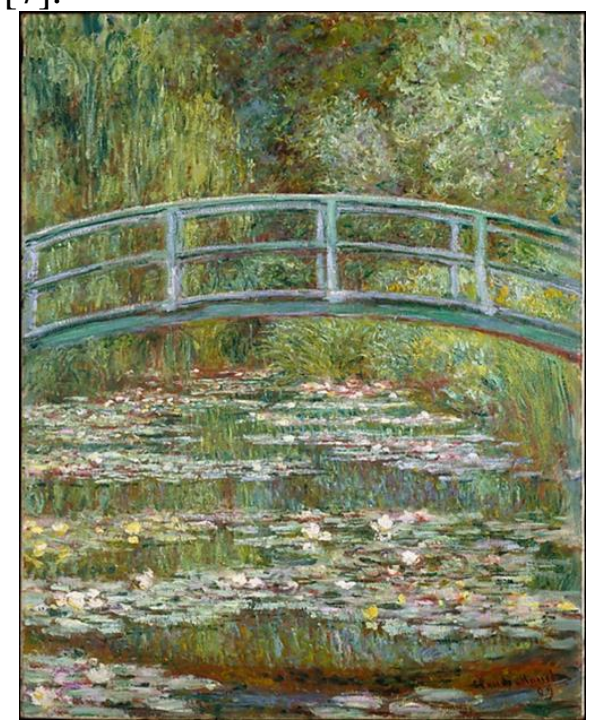

Figure 3. Claude Monet, 1899, Bridge over a Pond of Water Lilies. Oil on canvas, 92.7cm x 73.7 $\mathrm{cm}$. Acknowledgment: Open Access at the Met, Metropolitan Museum of Art [8].

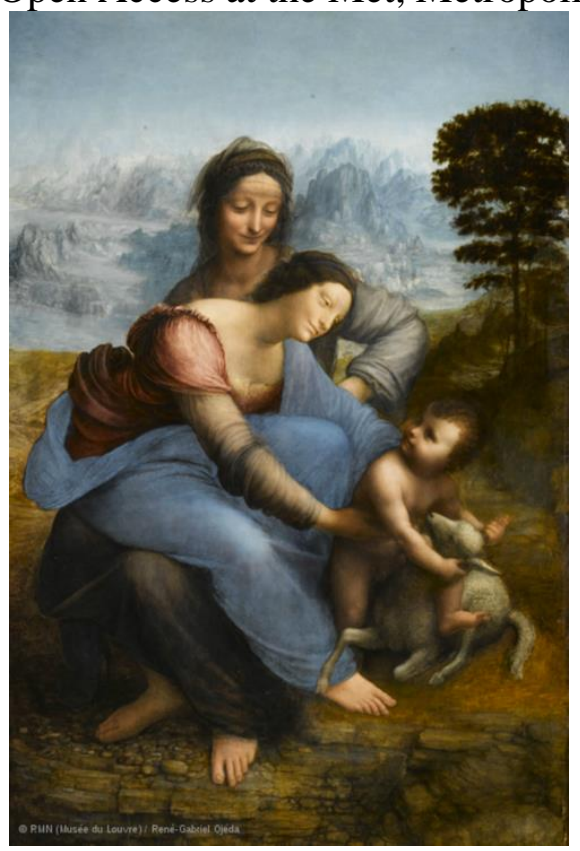

Figure 4. Leonardo Da Vinci, 1503 - 1519, Saint Anne, the Virgin Mary and the Infant Jesus, 1503-

1519. Oil on canvas, $1.68 \mathrm{~m}$ X 1.30m. Acknowledgment: Louvre Museum [9].

Figure 3 and 4 are a simple comparison of two images, one is the Impressionistic master, Claude Monet and the other is by the Renaissance master Leonardo da Vinci. The color scheme of both images are mapped and comparison of the data are shown below. 


\section{Results and Discussions}

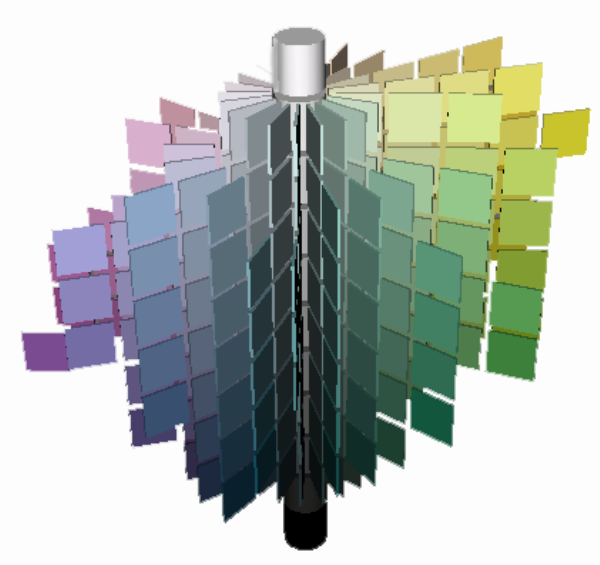

5 (a)

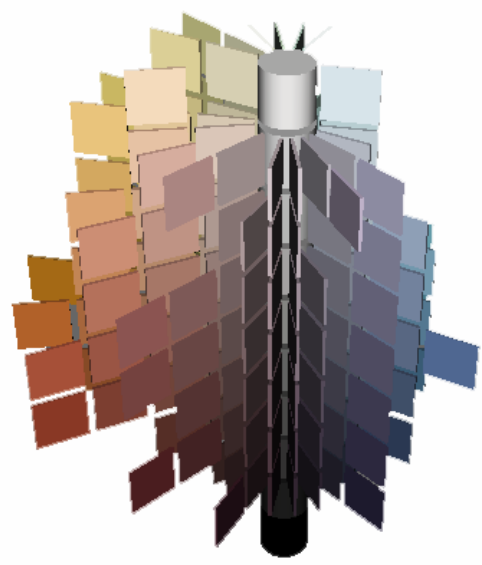

5 (b)

Figure 5 (a) and (b) are 3D models of plotted based on analytical results of Figure 3, (a Monet) and Figure 4 (a Da Vinci) respectively.

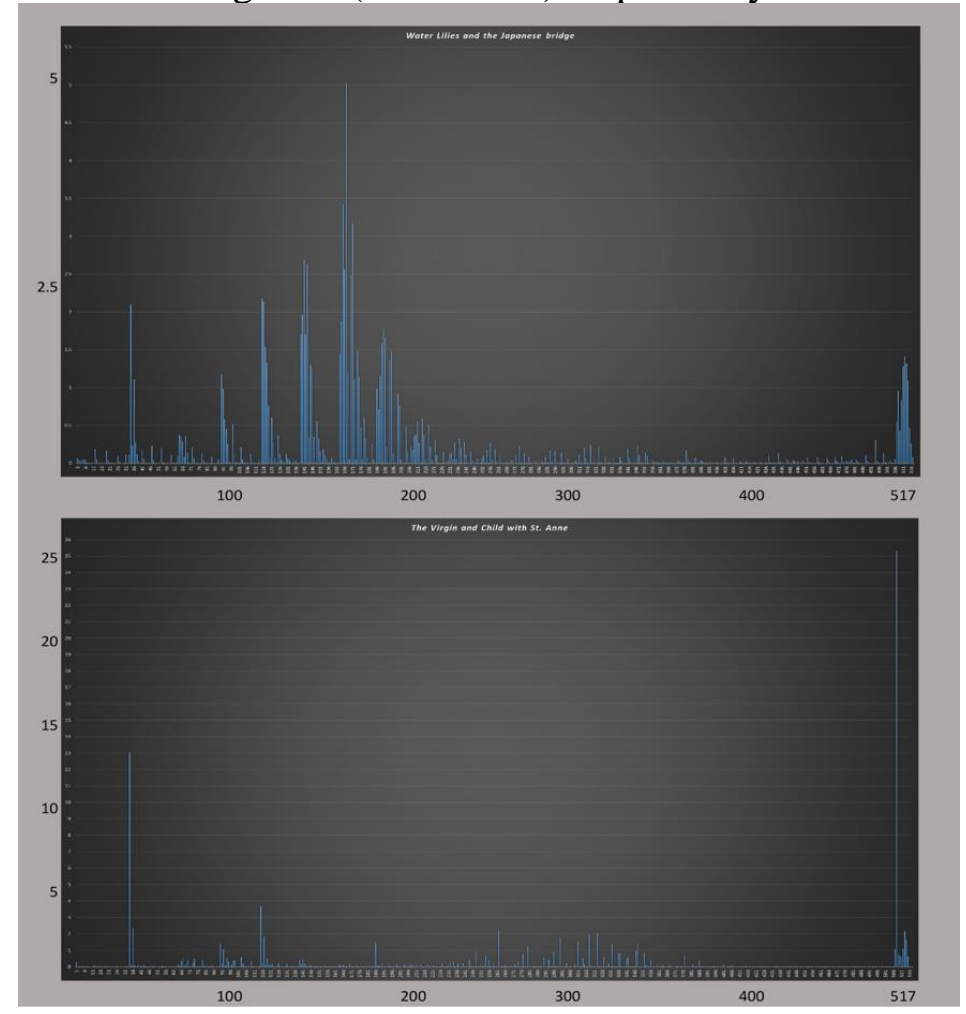

Figure 6. A Comparison between the color distribution of the Monet (top) and Da Vinci (bottom). Each color is represented by a number from 1 to 517 . There are total 517 colors plotted to form the Munsell Color Space.

The results help students visualize the difference between the two masterpieces from two different eras immediately. Figure 5(a) and (b) clearly indicates that Monet used colors stretch wider with the Munsell Color Space as compared to Da Vinci. Students can further visualize that the chroma of colors in Monet is higher, while the Da Vinci shows mainly browns and gray. The difference has been discussed in introduction due to the availability of painting pigments before and after the Industrial Revolution.

The histograms in Figure 6 further indicate the difference in color abundance for the two paintings. The Monet shows a lot more colors in therefore smaller abundances than the Da Vinci within the 517 colors categorized by the Munsell Color Space. These data can assist students in learning why Da Vinci used fewer colors and therefore leading to the learning of how Da Vinci achieved color riches with mostly browns and grays. In the end, students also learn about how Monet could manage many 
chromatic colors while he was still able to allow the colors to create image unity. This will not be further discussed as it is out of the scope of this project.

\section{Conclusion and Recommendation}

The software developed serve both visualization and analytical purpose in assisting students' learning of color theory. It also significantly reduces the time needed for analyzing colors of an artwork. It may also be used to further motivate students to look into the reasons of why and how colors were used in certain manner during different periods in the art history. However, there are also a few improvements need to be made to enhance the visualization and reusability of the software.

The imported 3ds Max for Java program to present the 3D model could not fully support the visual presentation because of its built-in presentation of 3D model that carries shadows. The shadows block certain color swatches which are supposed to be shown with their correct attributes. This may mislead students when the model is viewed as reference though the actual data analysis will not be affected. Therefore, it is better to model the 3D structure from scratch.

The presentation through histograms to show the color abundance and number of colors use is a good way to showcase the color scheme of an analyzed image, however, an improved graphical presentation will serve the purpose of reading the color abundances better. The higher abundance colors can be indicated clearly by graphical mean (the exact color) so that the end users can visualize major proportion of colors used in an analyzed image.

To allow reusability, a shared database could be built so that the analysis could be categorized and grow in size for reference. A standardize number of artworks can be first added as an initial reference library. Through looking at the built-in library of datasets, students could connect the history of color application with history of art better even before using the software for other image analysis.

\section{Acknowledgements}

This project was initiated by Information, Engineering and Media (IEM) program under School Electrical and Electronic Engineering (EEE) at Nanyang Technological University. The student and second author of this paper Mr. Xiao Yikai carried this software development as his final year project supervised by Assistant Professor Ng Woon Lam, the first author from School of Art, Design \& Media (ADM), NTU and Associate Professor Tan Yap Peng from EEE. The gratitude must go to Professor Tan for his time, patience, advice and efforts which have been of great help to Mr. Xiao's software development process. The gratitude also goes to both EEE and ADM for supporting this collaborating effort.

\section{References}

[1] Ng, Woon Lam, A New Approach for The Teaching of Practical Color Theory. (ICCI 2015 International Conference on Creative Industry; 2015 Aug 2015: ITS Press; 2015.)

[2] Westland, S., Laycock, Kevin., Cheung, Vien., Henry, Phil., Mahyar, Forough (2007). Color Harmony, Colour Journal - Design and Creativity 1(1): pp7.

[3] Gude, O. (1999). Color Coding, Art Journal 58(1): pp. 21-26.

[4] Ng, Woon Lam, Practical Applications of Color Theory and Design Concepts, Published by Author, Jan 2016, pp. 12

[5] Munsell, A. H., \& Farnum, R. B. A Color Notation: An Illustrated System Defining All Colors and Their Relations 1941. Baltimore, MD: Kessinger Publishing LLC (1941).

[6] Paul Centore, Conversions Between the Munsell and sRGB, USA 2013, http://www.munsellcolourscienceforpainters.com/ConversionsBetweenMunsellAndsRGBsyste ms.pdf. 
[7] Lyon, C. (1991). Unveiling Monet, MoMa 7: pp14-23.

[8] Monet, Claude. (1899). Bridge over a Pond of Water Lilies, Open Access at the Met, Metropolitan Museum of Art

[9] Da Vinci, L. (1503 - 1519). Saint Anne, the Virgin Mary and the Infant Jesus: Leonardo di Ser Piero DA VINCI, known as Leonardo da Vinci (Vinci, 1452 - Amboise, 1519), Saint Anne, the Virgin and the Child playing with a lamb, called La Sainte Anne. CA. 1503 - 1519, Acknowledgment: Louvre Museum, authorized for non-commercial re-use of this image. 